\title{
Encuadres del cambio climático en Chile: Análisis de discurso en prensa digital
}

Framing climate change in Chile: discourse analysis in digital media Julio Octavio Hasbún-Mancilla / jhasbun@med.uchile.cl http://orcid.org/0000-0001-5805-3277 Universidad de Chile, Chile Paulina Paz Aldunce-Ide / paldunce@uchile.cl http://orcid.org/0000-0002-1159-9333

Centro de Ciencia del Clima y la Resiliencia, Chile Gustavo Blanco-Wells / gblanco@uach.cl http://orcid.org/0000-0002-4980-3424 Rodrigo Browne-Sartori / rodrigobrowne@uach.cl http://orcid.org/0000-0001-8945-1059 Universidad Austral de Chile, Chile

\begin{abstract}
This article presents a discourse analysis of four digital media press in Chile with regard to mitigation and adaptation to climate change. The research, unprecedented for the Chilean case, is aimed at acknowledging the news framings by means of which climate change is communicated, since the media are the main source of information on climate change for decision makers and citizens. The results show that the primary definers of the topic are the governmental actors of national level and the invisibilization of individuals and civil organizations in the process. Thus, we see a high degree of consensus between the visible actors with regard to the framing of economic opportunity and the absence of framings of critical ecology. The conclusions point that this imbalance might influence a design of public policies with a technocratic bias, losing the possibility of building an integral vision of the development of the country.
\end{abstract}

Key words: climate change, communication, framing, adaptation, mitigation.

Resumen: Este artículo presenta un análisis de discurso a cuatro medios de prensa digital en Chile respecto a la mitigación y la adaptación al cambio climático. La investigación, sin precedentes para el caso chileno, se orienta a conocer los encuadres noticiosos con que es comunicado el cambio climático, ya que los medios son la principal fuente de información del cambio climático para los tomadores de decisión y la ciudadanía. Los resultados muestran que los definidores primarios del tema son los actores gubernamentales de nivel nacional, y la invisibilización de las personas y organizaciones ciudadanas en el proceso. Vemos así un alto grado de consenso entre los actores visibilizados respecto al encuadre de oportunidad económica, y la ausencia de encuadres de ecología crítica. Las conclusiones apuntan a que este desbalance podría influir en un diseño de políticas públicas con un sesgo tecnocrático, perdiendo la posibilidad de construir una visión integral del desarrollo del país.

Palabras clave: cambio climático, comunicación, encuadres, adaptación, mitigación. 


\section{Introducción $^{1}$}

\section{Cambio climático, desafio de política pública}

La voluntad política de los países democráticos depende en gran medida de la tabla de prioridades que existen en la agenda pública en un momento dado, razón por la cual el poder que poseen los medios de comunicación es fundamental, gracias a su capacidad para influir en la opinión pública, constructo medible gracias a encuestas de opinión periódicas (Kingdon, 1995: 90).

Entendemos al cambio climático no como fenómeno natural sino eminentemente político, al versar no sólo en las transformaciones provocadas por el hombre en los diversos sistemas de sostenimiento de la vida, sino además en los procesos de toma de decisiones, por cuanto requiere de altos niveles de coordinación de acciones y múltiples innovaciones en la forma de entender y diseñar la política pública (Giddens, 2010: 15).

En esta dirección, el brasileño Achim Steiner (2013), director ejecutivo del Programa de Naciones Unidas para el Medio Ambiente ha planteado: "El reto que enfrentamos no es técnico ni de normas. Es político: el ritmo actual de acción es insuficiente", respecto a la posibilidad de limitar el aumento de la temperatura del planeta en $2^{\circ} \mathrm{C}$.

Una manera de aproximarnos al proceso de construcción de agendas es la noción de encuadre, ya que permite configurar los límites de un debate al establecer un número acotado de alternativas, que serán posibles de observar para los tomadores de decisión al momento de comprender, planificar y gestionar un problema de política pública (Pralle, 2009: 783).

\section{Latinoamérica y Chile ante el cambio climático}

Así como el cambio climático no impacta de igual forma a distintas latitudes y territorios, posee efectos diferenciales al interior de cada sociedad, lo cual para el caso de Latinoamérica se traduce en que golpea con mayor fuerza a aquellos que son económica, política, social y culturalmente más vulnerables, motivo por el cual la política de cambio climático puede ser considerada en este sentido como una política de desarrollo, pues mitigar sus impactos involucra necesariamente elevar las condiciones de vida de la población (Giddens, 2010: 20; Cannon y Müller-Mahn, 2010: 620).

1 Investigación que contó con el financiamiento y en el marco del proyecto FONDAP \#151 1009, “Centro de Ciencia del Clima y la Resiliencia”: Universidad de Chile, Universidad Austral de Chile, y Universidad de Concepción, Chile. 
Esto es relevante por cuanto existe un desbalance entre los hemisferios norte y sur respecto a la cantidad de: i) producción científica (IPCC, 2014: 842), y ii) producción de noticias de cambio climático (Schmidt et al., 2013: 1237) en favor del primero, generando sesgos y distorsiones en la comprensión del fenómeno por parte de nuestras sociedades, pues la investigación de las últimas tres décadas muestra en forma consistente que el público general, y los tomadores de decisión como parte de dicha audiencia, acceden a comprender la ciencia y el cambio climático fundamentalmente a través de los medios de comunicación ${ }^{2}$ (Boykoff y Yulsman, 2013: 360).

El importante crecimiento económico de la última década en Latinoamérica ha generado que muchos de nuestros países sean considerados como de ingreso medio, los que enfrentan disyuntivas críticas de cara al cambio climático, pues deben hacer frente a preguntas de difícil respuesta técnica y política: ¿enfrentamos la vulnerabilidad actual o la futura?, o bien, ¿ ponemos nuestro foco en la adaptación o en la mitigación al cambio climático? Hay evidencia que indica que los países de ingreso medio son más vulnerables a los impactos del cambio climático (sequía, seguridad alimentaria, entre otros) que los más pobres, en el dilema adaptación versus desarrollo, pues el periodo de transición desde modos de vida tradicional a otros modernos implica no sólo ganancias, sino pérdidas (Fraser et al., 2013: 199).

La capacidad de adaptación al cambio climático en Latinoamérica es baja (IPCC, 2014: 1537), y aun cuando nuestra contribución a la emisión de gases de efecto invernadero es pequeña, igualmente debemos considerar su mitigación. Además, es reconocida la limitada capacidad de nuestros Estados para: $i$ ) hacer frente a tensiones y procesos combinados, como los involucrados en el cambio climático; ii) el bajo número de políticas nacionales que integran variables de cambio climático; y iii) las dificultades para solucionar la raíz de los problemas, cuando los tomadores de decisiones no tienen entrenamiento o información suficiente, en contextos de restricción presupuestaria fiscal y en presencia de conflictos a distintas escalas del gobierno (Hardoy y Pandiella, 2009: 220).

En este escenario, Chile destaca por ser el primero que aplicó las políticas del recetario neoliberal, generando altos grados de desigualdad (económica, política, geográfica, ambiental), razón por la que es relevante analizar los encuadres noticiosos del cambio climático, pues permitirá aproximarnos al proceso de construcción de las agendas pública y política, con el fin de comprender cuál es el horizonte de posibilidades que pueden visualizar las personas al momento de pensar y actuar frente al cambio climático.

2 Como la televisión, diarios, revistas, radio, noticias en línea, sitios agregadores de contenidos, blogs y medios sociales. 


\section{Concentración de medios en Latinoamérica}

Un denominador común en Latinoamérica es la alta concentración en la propiedad de sus medios de comunicación, con importantes consecuencias para el requisito de pluralidad de información que sustenta a los Estados democráticos, pues éstos tienen la función de observar y controlar el ejercicio del poder en las democracias (Sunkel y Geoffroy, 2001: 13).

La privatización de los años noventa (con la excepción de Uruguay), justificada para hacer ingresar más actores y generar mayor competitividad, no cambió en la práctica el panorama mono u oligopólico que presentaban las industrias infocomunicacionales de Latinoamérica, siendo la concentración más elevada en países pequeños como Chile, Perú y Uruguay, con la radio como el sector menos concentrado y la telefonía como el más concentrado (Mastrini y Becerra, 2006: 117).

En esta dinámica, ha sido el mercado quien ha fijado las principales estrategias del sector en Latinoamérica, "para que con posterioridad el Estado ajustara el marco regulatorio a dicha situación” (Mastrini y Becerra, 2006: 307). El caso chileno no escapa a dicha lógica, profundizándola, pues la política de medios posterior a la dictadura cívico-militar fue: "la mejor política es no tener política” (Sunkel y Geoffroy, 2001: 12), donde la no intervención estatal se tradujo en la desaparición de diversos medios creados a fines del gobierno militar, situación que llevó a la ONG Human Rights Watch (1998: 49) a señalar que la libertad de expresión e información en Chile estaba limitada "hasta un nivel posiblemente incomparable con cualquier otra sociedad democrática del hemisferio occidental”.

La concentración de la propiedad de los medios posee tres consecuencias sobre la libertad de expresión: i) la subordinación de los medios al poder económico; ii) el debilitamiento de la cultura profesional de los periodistas; y iii) que los medios no se constituyen en canales de expresión de la ciudadanía. Para el caso de Chile, estos procesos van acompañados de un "monopolio ideológico", donde la diversidad cultural y política fue relegada a un plano marginal por la industria de la entretención (Sunkel y Geoffroy, 2001: 114-115).

En este panorama, cuando el debate se orienta hacia temas de alta complejidad -con importantes riesgos para la seguridad humana como el cambio climático-, los efectos de dichos procesos pueden ser potencialmente catastróficos. 


\section{Discursos mediales del cambio climático}

El rol central de los medios en el proceso de construcción de las agendas pública y política en torno al cambio climático ha motivado el análisis del discurso medial, especialmente en los países industrializados (Schmidt et al., 2013: 1239), con excepciones como el caso de India (Jogesh, 2012: 266), Brasil (Painter y Ashe, 2012: 2) y Perú (Takahashi y Meisner, 2013: 340). En la larga historia de la investigación del rol de los medios en la cobertura a temas ambientales, no es sino hasta la década de 1990 cuando la atención se focaliza sobre el cambio climático (Anderson, 2009: 166), entrando en la agenda como un tema fuertemente politizado, en especial en Estados Unidos e Inglaterra (Boykoff y Boykoff, 2004: 125), pasando desde un control discursivo en manos de los científicos climáticos hasta los políticos, luego del "discurso verde" que dio Margaret Thatcher a la Royal Society durante 1988 (Anderson, 2009: 168).

En el primer lustro de la década de 1990, el tema pierde fuerza al competir con tópicos como la crisis económica y la guerra de Irak, para en 1997 volver a emerger gracias al Protocolo de Kioto, esta vez acompañado de nuevos estudios respecto a sus impactos en los países desarrollados (Boykoff y Boykoff, 2007: 1190).

Los primeros años de la década del 2000 siguió acaparando la atención de los medios en Estados Unidos e Inglaterra (Boykoff, 2007: 1198), especialmente durante el 2006, asociado al documental de Al Gore, "Una verdad incómoda".

Irónicamente, ha existido una muy baja cobertura del cambio climático en los países en desarrollo en términos comparativos, aun cuando son los que sufren sus peores efectos (Painter y Ashe, 2012: 6).

Desde la interfaz entre comunicación y política, este artículo busca comprender cómo se comunica la mitigación y adaptación al cambio climático en Chile, mediante un análisis de discurso a cuatro medios de prensa digital, durante el periodo 2011-2013.

La interrogante que guió esta investigación es: ¿cómo los medios digitales presentan la mitigación y adaptación al cambio climático en Chile?, con el fin de aproximarnos a los procesos de construcción de las agendas pública y política, gracias al análisis de los encuadres noticiosos en la prensa digital. 


\section{Marco conceptual}

Se analizaron los encuadres noticiosos del cambio climático en Chile, que pueden definirse como "la idea organizadora central para el contenido de las noticias, que provee un contexto y sugiere qué es el tema mediante el uso de la selección, énfasis, exclusión y elaboración” (Ryan et al., 1991: 3). Así, los encuadres orientan la perspectiva con que son narradas las noticias, generando narrativas que, amplificadas en el espacio público por los medios, aportan a la definición y construcción de las visiones de mundo y estilos de vida que sustentan los individuos, sin desmerecer la capacidad de agencia e interpretación que éstos poseen. Los encuadres noticiosos son importantes por su habilidad para "definir los términos del debate sin que la audiencia lo note" (Tankard, 2001: 97). Al recortar la realidad, los encuadres noticiosos son instrumentos de poder y control social, ya que los actores poseen un acceso diferencial en sus procesos de diseño y comunicación.

Hall et al. (1978: 647) observan las estructuras rutinarias de la producción de noticias, concluyendo que en última instancia los medios reproducen las definiciones de los poderosos, en tanto fuentes acreditadas. Así, plantean que la política es el definidor primario que configura y encuadra "lo que el problema es". Este encuadre inicial provee los criterios bajo los cuales todas las contribuciones subsiguientes son consideradas como "relevantes" o "irrelevantes".

Desde esta mirada, sería la agenda política la que definiría la agenda de los medios, los cuales amplificarían estas definiciones ya encuadradas hacia el público, definiendo también, a su vez, los temas prioritarios (CervantesBaraba, 2001). Gandy (1989: 270) señala que "ciertas clases de fuentes han sido identificadas como más confiables que otras. Las burocracias oficiales, o las instituciones organizadas burocráticamente, tienden a ser las más confiables, y como resultado, la información provista por las burocracias tiende a dominar los canales de los medios".

Desde esta óptica, es relevante considerar además a empresarios y grupos de interés poderosos, que también tendrían capacidad de construcción de la agenda medial. Así, los medios se encontrarían en una subordinación estructurada frente a los definidores primarios (Hall et al., 1978: 650).

La teoría de la fijación de la agenda permite comprender la vinculación entre opinión pública, grupos de presión, medios de comunicación y tomadores de decisión, al intentar responder a la pregunta de por qué ciertos temas emergen en las agendas gubernamentales, mientras otros son descuidados (Kingdon, 1995: 7). 
Los problemas surgen -obtienen saliencia- y desaparecen de las agendas políticas y públicas, en forma independiente de su estado objetivo (Baumgartner y Jones, 1993: 142), y problemas que no poseen soluciones disponibles o factibles; incluso si atraen la atención del público y el gobierno, es probable que no lleguen a la agenda de decisión (Kingdon, 1995: 44).

\section{Marco metodológico}

El análisis de contenido cuantitativo permite computar y sistematizar la información, ${ }^{3}$ para generar inferencias objetivas de la frecuencia de emergencia y uso de ciertos objetos de análisis, que en segunda instancia posibilitarán realizar un análisis de discurso sobre dicho procesamiento.

El problema que guía esta investigación se aproxima al campo de estudios denominado "ciencia y política", interfaz compleja donde los conceptos de conocimiento y poder confluyen. Desde esta óptica, el dispositivo de análisis más pertinente es el Análisis Crítico de Discurso (ACD), que es el utilizado en esta investigación.

Van Dijk (2002: 4) orienta la perspectiva que toma el ACD ante el discurso, al poner el acento en los problemas sociales prevalentes, eligiendo "el punto de vista de los que más sufren, y analizando críticamente aquellos en el poder, los que son responsables, y los que tienen los medios y la oportunidad de resolver tales problemas".

Vemos así que en los textos convergen diferencias discursivas que son negociadas y gobernadas por el diferencial de poder que cada una posee, lo cual en ocasiones hace que éstos sean sitios de lucha y control, mostrando trazas de las ideologías en conflicto, pues el poder descansa en relaciones de diferencia $y$, particularmente, en los efectos de las diferencias en las estructuras sociales.

En este artículo analizamos los encuadres noticiosos utilizados para presentar el cambio climático, los cuales se constituyen como narrativas que poseen grados mayores o menores de institucionalización. Cuando estas narrativas expresan posiciones conflictivas, producto del enfrentamiento entre quienes aspiran a cambiar las estructuras sociales y aquellos que tienen intereses para mantenerlas, toman la forma de discursos, en tanto dispositivos de control para el acceso diferencial a decidir respecto al uso de ciertos recursos (económicos, políticos).

3 Realizado mediante el uso del programa de análisis de datos no estructurados Nvivo 10. 


\section{Unidad de análisis}

La unidad de análisis es la noticia, que en los medios de comunicación es un reporte formal de eventos considerados como significativos para la audiencia objetivo, normalmente publicados poco tiempo después de que su información llega a estar disponible (Chandler y Munday, 2011: 227). Se espera que sea una comunicación informacional referencial y objetiva, sin sesgos, aun cuando la selección de la información está determinada por los valores noticiosos, es decir, por los criterios periodísticos informales adoptados por la selección, priorización y presentación de los eventos por parte de la línea editorial del medio.

\section{Muestra}

La muestra considerada son medios de prensa digital. En Chile, la prensa digital cuenta con un nivel similar de credibilidad que la impresa, es percibida como más independiente para informar, y con un nivel similar de calidad entre ambas. Es el cuarto medio de comunicación más utilizado en el país, luego de la televisión abierta, la radio y la televisión por cable, superando levemente a los diarios impresos (UDP-Feedback, 2011: s/p). Su alcance temporal abarca la Segunda Comunicación Nacional de Cambio Climático (30 de agosto de 2011) y la creación del Centro de Ciencia del Clima y la Resiliencia (31 de octubre de 2013), dado que es un periodo relevante en términos del posicionamiento que estaba obteniendo el tema en la agenda nacional.

Para conocer la diversidad de tendencias presentes en el discurso público, se han seleccionado cuatro medios de prensa digital (La Nación, El Mostrador, La Tercera, El Mercurio), tanto por su perfil político-editorial (centroizquierda/ derecha), como por su nivel de lectoría (Tabla $1^{4}$ ). El duopolio existente en los medios de prensa escrita (impresos y digitales) se muestra en los niveles de concentración de lectoría que poseen los medios conservadores por sobre los liberales (Sunkel y Geoffroy, 2001: 17).

Sobre el corpus de noticias obtenido, se realizará una codificación de la información en dos nodos centrales: "mitigación del cambio climático" (MCC) y "adaptación al cambio climático" (ACC), sobre los cuales, a su vez, se hará una codificación de segundo orden en distintos encuadres (subnodos), con el fin de facilitar el análisis de discurso.

4 Las tablas se encuentran en el Anexo, al final del presente artículo (Nota del editor). 


\section{Criterios de selección}

Los criterios de selección de la muestra son los siguientes:

1. Inclusión de noticias nacionales, incluyendo de chilenos en el extranjero hablando del país o bien de extranjeros hablando de Chile. Se excluyen editoriales, columnas de opinión y entrevistas, ya que su lógica no responde a la estructura del estilo noticioso.

2. Noticias que en su título, bajada (subtítulo), primer y segundo párrafo mencionen alguno de los siguientes conceptos: i) cambio climático, ii), calentamiento global, iii) cambio global y iv) efecto invernadero.

\section{Resultados}

Mediante la aplicación de los criterios de selección señalados en el marco metodológico, se obtuvo un corpus de 58 noticias de cambio climático en Chile durante el periodo analizado, sobre las que se realizará el análisis de discurso. Si tomamos el conjunto de los cuatro medios estudiados, encontramos con que se publica en promedio una noticia nacional de cambio climático cada 12,78 días. El medio electrónico El Mostrador no presenta noticias nacionales de cambio climático, ya que toma como fuente a agencias internacionales.

Los actores con mayor presencia son los políticos, seguidos por los científicos y expertos, y los organismos internacionales. Más atrás están los empresarios, siendo notable la ausencia de actores ciudadanos (Tabla 2).

Respecto a cada nodo y subnodo analizado, se observa un número equivalente de noticias en relación con la mitigación $(\mathrm{N}=28)$ y la adaptación $(\mathrm{N}=29)$ al cambio climático en Chile.

Para el análisis, se ha definido la MCC como: "una intervención humana para reducir las fuentes o mejorar los sumideros de gases de efecto invernadero". Desde la perspectiva del Panel Intergubernamental para el Cambio Climático $\left(\right.$ IPCC $\left.^{5}\right)$, al ser éste causado por la acción humana, fundamentalmente por la quema de combustibles fósiles que liberan gases de efecto invernadero (GEI) como el CO2, es posible realizar distintas actividades de mitigación que contribuyan a disminuir la cantidad de dichos gases en la atmósfera.

La MCC ha sido subdividida para el análisis en cuatro encuadres: i) "reducción de intensidad de emisiones", ii) "reducción absoluta de emisiones”, iii) “metas de carbono neutralidad” y iv) “otras acciones de mitigación”.

5 IPCC, por sus siglas en inglés. 
Casi la mitad de las noticias estudiadas $(28 / 58)^{6}$ contiene información referente a la MCC, ya sea con menciones implícitas o explícitas. La mayor cantidad de noticias se orienta al encuadre "otras acciones de mitigación" (12), seguida a continuación por la de "reducción de intensidad de emisiones" (10), "metas de carbono neutralidad" (5) y "reducción absoluta de emisiones", que no posee referencias.

De los cuatro encuadres analizados, tres de ellos presentan noticias en tres de los medios digitales revisados que contienen noticias de cambio climático en Chile, siendo los medios con mayor número de noticias La Tercera y El Mercurio (ambos con 9), seguido más atrás por La Nación, con dos noticias.

Por otra parte, la ACC se ha definido como: "ajustes en sistemas humanos o naturales como respuesta a estímulos climáticos proyectados o reales, o sus efectos, que pueden moderar el daño o bien aprovechar sus aspectos beneficiosos". Este nodo ha sido subdividido en cuatro encuadres: $i$ ) "planificación", ii) "vulnerabilidad", iii) "riesgo económico" y iv) "medidas existentes", tomando como referencia los encuadres hallados por Juhola et al. (2011: 456).

En la mitad de las noticias analizadas (29/58) se aprecia información referente a la ACC, en forma implícita o explícita. La mayor cantidad de noticias se orienta al encuadre "planificación" (20), seguido a continuación por los de "vulnerabilidad" y "riesgo económico" (ambos con 15), poniendo en último lugar el de "medidas existentes" (8). De los cuatro encuadres analizados de ACC, tres de ellos presentan noticias en tres de los medios digitales revisados que contienen noticias de cambio climático en Chile, y sólo en el caso del encuadre "vulnerabilidad" no encontramos referencias de El Mercurio, aun cuando es el medio de la muestra con el mayor número de noticias de cambio climático en Chile.

\section{Discusión}

\section{¿Mitigación o adaptación al cambio climático?}

$\mathrm{Al}$ ser Chile un país sudamericano en vías de desarrollo, con un ingreso medio y miembro OCDE, se encuentra en un interregno: no es un país rico y desarrollado, ni tampoco uno en situación de pobreza, lo cual complejiza el proceso de decisión en la disyuntiva mitigación/adaptación. En general, se aconseja que los países desarrollados inviertan principalmente en mitigación,

6 Este número (28 noticias) no considera el fenómeno de yuxtaposición, por el cual una noticia en particular puede contener referencias a distintos encuadres. En adelante los números presentados responden al mismo fenómeno. 
y los países en desarrollo, en adaptación, básicamente por el uso intensivo que hacen los primeros de combustibles fósiles, y porque los segundos deben invertir menos para acortar brechas con los países desarrollados y entregar así mejores condiciones de vida a sus habitantes, en lo que Giddens (2010:17) ha denominado "imperativo de desarrollo".

En esta dinámica los países en desarrollo hacen una adaptación reactiva, dado que no disponen de recursos propios para hacer frente a los impactos proyectados, y la cooperación internacional y la transferencia tecnológica juegan un rol fundamental para apoyar el paso hacia una adaptación planificada.

Para ser efectiva la mitigación, debe ser realizada a escala global; en cambio, la adaptación es más efectiva a escala de un sistema impactado, a nivel local y regional. La mitigación posee una métrica establecida; en cambio, conocer los beneficios de la adaptación depende de contextos sociales, económicos y políticos (Broomell et al., 2015: 72). Los beneficios de la mitigación serán evidenciados en muchas décadas más, por la larga residencia de los GEI en la atmósfera; mientras los de la adaptación son efectivos en el presente, al reducir la vulnerabilidad a la variabilidad climática. Y a medida que el cambio climático continúe, los beneficios de la adaptación se incrementarán en el tiempo. La pregunta que captura el problema apunta a: ¿cuáles combinaciones de reducción de emisiones y adaptación pueden mitigar en mejor forma los impactos del cambio climático?

El Panel Intergubernamental para el Cambio Climático, al estudiar las interrelaciones entre adaptación y mitigación, plantea que existen disyuntivas y sinergias entre ambas medidas, dado que la una tiene consecuencias para la otra, razón por la cual deben ser diseñadas para aprovechar las complementariedades, reduciendo sus interferencias negativas (IPCC, 2014). Ayers y Huq (2008: 757) señalan los beneficios de integrar ambos enfoques a nivel de proyecto en Bangladesh, afirmando que va más allá de alinear los incentivos, ayudando a conseguir respaldo a la adaptación entre los defensores de la agenda "fuerte" de mitigación, que han sido cautelosos con la adaptación en el pasado.

Somorin et al. (2012: 292), en su análisis de discurso a las políticas de respuesta al cambio climático en el sector forestal del Congo, hallaron tres discursos: políticas sólo de mitigación; políticas separadas de adaptación y mitigación; y políticas integradas de adaptación y mitigación.

En este último discurso los encuadres encontrados apuntan a que: i) existen ventanas de oportunidad para las sinergias; ii) es posible diseñar una medida para integrar a la otra; iii) aparentemente poseen marcos institucionales y jurídicos similares para su diseño e implementación; y iv) el hecho 
que comparten una política de resultados para la reducción de la pobreza, la conservación de la biodiversidad y el desarrollo. Es relevante destacar que las coaliciones en competencia tras los tres discursos señalados subrayan sus posiciones e intereses con elementos financieros, de poder, control, conocimiento, influencia y justicia.

Los resultados de esta investigación muestran que la prensa digital revisada expone la mitigación y la adaptación sin ahondar en los beneficios y costos de la aplicación de una u otra estrategia. Que no se definan en forma clara ambos términos puede estar asociado al hecho de que, en general, pocas noticias de ciencia incluyen información acerca del proceso científico (Alley, 2012: 174; Arcila-Calderón et al., 2015: 86), y el caso chileno no es la excepción, ya que no se explicitan los fundamentos bajo el cual adquieren sentido las acciones de mitigación y adaptación (Jang y Hart, 2015: 16).

Es importante notar que la estructura del formato "noticia" no alcanza en general más allá de las 500 palabras en promedio (Sterman, 2011: 817), por eso existe una dificultad para comunicar los contextos de sentido de los eventos presentados, ya que se privilegia entregar la mayor cantidad de información en el menor espacio posible, lo cual juega en contra de temáticas complejas. A esto hay que añadir el bajo nivel de comprensión lectora de los adultos chilenos, ${ }^{7}$ el muy bajo nivel de escolaridad, ${ }^{8}$ y el bajo consumo de literatura en general, ${ }^{9}$ situación que Sterman (2011: 816) también destaca para el caso del público estadounidense, existiendo una brecha entre el entendimiento de las ciencias básicas y los Resúmenes para Responsables de Políticas, ${ }^{10}$ que requieren al menos 17 años de estudio para ser comprendidos.

7 El Centro de Microdatos de la Universidad de Chile (2013) señala que el analfabetismo funcional alcanza al 44\% de la población adulta del país, cifras idénticas a las medidas en 1998 .

85,2 millones de personas mayores de 18 años no han terminado la enseñanza secundaria en Chile (42,9\%) (Melo, 2013).

9 Un 31,3\% de la población compra en promedio un libro al año. Encuesta Nacional de Participación y Consumo Cultural. Análisis Descriptivo. (Consejo Nacional de la Cultura y las Artes, 2012).

10 Textos de síntesis para los tomadores de decisión editados por el Panel Intergubernamental para el Cambio Climático. 
Julio Octavio Hasbún-Mancilla, Paulina Paz Aldunce-Ide, Gustavo Blanco-Wells y Rodrigo BrowneSartori. Encuadres del cambio climático en Chile: Análisis de discurso en prensa digital

\section{Mitigación}

En la muestra analizada, la mitigación se presenta, principalmente, a nivel transnacional, como reuniones y conferencias internacionales (por ejemplo, la XVII Conferencia de las Partes de la Convención Marco de Naciones Unidas sobre Cambio Climático, COP 17) y a nivel nacional, tanto como respuesta del gobierno central (desarrollo de modelos de mitigación), como por los esfuerzos realizados por actores empresariales (Corporate Leaders Group Chile), consistente con lo hallado por Olausson (2009: 430) al analizar tres diarios suecos.

Las acciones de mitigación son usualmente desplegadas en el macronivel (nacional, transnacional); no existen noticias que conecten la mitigación con acciones individuales o den voz a organizaciones no gubernamentales asociadas al tema.

Ockwell et al. (2009: 320) afirman que la comunicación juega un rol fundamental en orden a que la mitigación sea efectiva, señalando que ésta posee dos roles: primero, facilitar la aceptación de regulaciones por parte del público (top-down), y segundo, estimular la acción de base mediante un compromiso afectivo y racional con el cambio climático (bottom-up). Señalan que sólo la combinación de ambas aproximaciones permitiría aumentar los niveles de involucramiento de las personas con el cambio climático, aportando lidiar con las barreras percibidas a nivel estructural-social y a nivel subjetivo-individual.

La forma de encuadrar la mitigación por parte de los medios de prensa digital estudiados se orienta a narrativas que ponen énfasis en la dimensión política, quedando en un plano bastante menor la científica, lo cual permite plantear que en la comunicación de la mitigación, en estos medios de prensa chilenos, es la política quien define los límites de la mitigación, tanto en forma explícita ("lo dicho") como implícita ("lo no dicho"). El mundo de las grandes corporaciones aparece asociado a medidas de carbono neutralidad, y las PYMES no emergen como fuentes ni mencionadas por los actores visibilizados. Mención aparte merecen las personas y actores del tercer sector (ONG, Fundaciones, entre otros), los cuales no tienen cabida en las noticias de mitigación en los medios estudiados.

En Chile se presenta un balance entre las noticias de adaptación y mitigación al cambio climático durante el periodo cubierto por el análisis, lo cual contrasta con lo sucedido en Perú, donde entre el 2000 y el 2010 se aprecia que la estrategia de adaptación (65\%) superó largamente a la de mitigación (17\%) (Takahashi y Meisner, 2013: 436). 


\section{Adaptación}

Vicuña (2012) señala que, de forma simple, es posible ver la adaptación como una reducción contextual (actual) o como resultado (futuro) de la vulnerabilidad. En esta disyuntiva, los países ricos deberían ocuparse de resolver la vulnerabilidad futura, y los pobres más de la actual, situación que para los países de ingreso medio como Chile se torna compleja, ya que deberían ocuparse de ambas, para lo cual debiesen buscar su confluencia. Chile comenzó su camino a la adaptación poniendo un fuerte énfasis en el desarrollo de conocimiento para lidiar con la vulnerabilidad presente, pero que en la actualidad estaría girando hacia la resolución de los problemas de vulnerabilidad actual.

Bassett y Fogelman (2013: 42) presentan un análisis de contenido que muestra el predominio (70\%) del enfoque de "adaptación de ajuste", por sobre el de "adaptación transformativa" (3\%). En el caso de las noticias analizadas, también se observa esta dominancia, donde se consideran los impactos climáticos como principal fuente de vulnerabilidad, sin prestar atención a sus raíces sociales ("adaptación transformativa"), de tal forma que el discurso de los medios no expresa la necesidad de generar transformaciones estructurales en la vida política, social e individual, y más bien expresan el punto de vista de reformas incrementales, las cuales aun cuando puedan conducir en el largo plazo a cambios en la conducta de los individuos, no sabemos si bastarán para evitar la degradación del medioambiente a puntos que permitan la sostenibilidad de las generaciones futuras.

La adaptación en la muestra analizada emerge principalmente como planificación, es decir, como búsqueda de soluciones a los impactos del cambio climático. En el caso de Inglaterra, esta narrativa pone el foco en la integración de las preocupaciones de adaptación en los procesos de planificación, con un énfasis en la revisión de las políticas existentes de planificación (Juhola et al., 2011: 456).

El caso chileno muestra un esperable menor nivel de maduración en la discusión de planificación, ya que actualmente se están dando los primeros pasos para generar políticas de adaptación, con énfasis tanto en la producción de información científica, como en encuentros de discusión nacional e internacional.

En segundo lugar en orden de relevancia, surge el encuadre de vulnerabilidad, donde la adaptación es vista como respuesta a la vulnerabilidad percibida a los impactos del cambio climático (Juhola et al., 2011: 456). En el caso de Suecia, este encuadre se encuentra conectado a eventos climáticos extremos como inundaciones. En Chile, en cambio, se observa a la vulnerabi- 
lidad como proyección de impactos futuros, es decir, no se percibe al cambio climático actuando en el presente. ${ }^{11}$ Nuevamente, no se profundiza en los aspectos sociales e históricos de la vulnerabilidad, lo cual va a influir en la manera como concibamos la adaptación, ya que "la política de adaptación será encuadrada de acuerdo a cómo el riesgo y la vulnerabilidad sean conceptualizados" (Bassett y Fogelman, 2013: 52).

Ambos elementos (desconexión de la vulnerabilidad y exposición actual con el cambio climático presente, y la desconexión con los aspectos sociales de la vulnerabilidad) generan: i) incomprensión de las audiencias respecto a quiénes son las poblaciones más expuestas y vulnerables al cambio climático, e ii) incomprensión de la necesidad de actuar disminuyendo la vulnerabilidad económica y social actual, al ser dichas poblaciones las más vulnerables a los impactos presentes y futuros del cambio climático.

En tercer lugar, aparece la narrativa de "riesgo económico", donde la adaptación es presentada como costos y riesgos económicos presentes y futuros (Juhola et al., 2011: 456), con un amplio respaldo en la política (8 actores), y emergiendo no sólo como costos sino también como oportunidades de negocio (7 noticias). Esta visión positiva de oportunidad económica apunta al encuadre de modernización ecológica, sustentado en las últimas décadas en Inglaterra por el gobierno del New Labour (Uusi-Rauva y Tienari, 2010: 496), que contrasta con la posición emergente en los medios asociados a la centro-izquierda chilena (lanacion.cl=1), que tienden a connotar los efectos negativos, como la pérdida de empleos e infraestructura.

Para Cannon y Müller-Mahn (2010: 622), es fundamental separar el concepto de "desarrollo" del de "crecimiento económico", que han sido confundidos desde la década de 1980 y que en la muestra analizada tienden a conjuntarse en la narrativa de "oportunidad económica”. Esta narrativa alberga en su interior la idea de que el crecimiento económico y acumulación de riqueza de los grupos más ricos eventualmente permeará hacia las capas inferiores de la sociedad, ${ }^{12}$ idea defendida por think tanks de derecha ${ }^{13}$ en Chile, fieles al modelo económico implantado por el gobierno militar durante los años ochenta, y que no ha podido ser contestada en forma efectiva por los gobiernos democráticos de las últimas décadas.

11 Por ejemplo, a través de las continuas y agudas sequías que vive el país, con un régimen pluviométrico que viene disminuyendo desde hace décadas en el centro y sur. Garreaud (2011) enumera los impactos observados en Chile durante el siglo XX y proyectados al XXI.

12 Conocida como "teoría del chorreo".

13 Los más importantes: "Libertad y Desarrollo”, "Centro de Estudios Públicos”, "Fundación Jaime Guzmán”. El discurso neoliberal afirma que la mejor forma de reducir la pobreza es mediante el crecimiento económico. 
Así, el crecimiento económico no es en sí adaptación al cambio climático, y sí lo es un concepto de "desarrollo con sentido", abocado directamente a mejorar la vida de las personas, que no sea un efecto secundario de lo que otros actores (corporaciones o gobiernos) hagan en su búsqueda de ganancia y crecimiento (Cannon y Müller-Mahn, 2010: 624-625). De esta forma se habla de un "sesgo económico" de los tomadores de decisión, donde los problemas son definidos exclusivamente como un asunto de calcular costos y beneficios (Dewulf, 2013: 327).

Estos sesgos se corresponden con la evasión de responsabilidad de las élites chilenas frente al tema, apuntando en sus discursos a: a) la limitada información provista por las agencias oficiales y los medios; b) falta de claridad de los científicos; c) medidas insuficientes a nivel institucional; $d$ ) causas y condiciones estructurales e institucionales; y e) responsabilidad del Estado y fallas regulatorias (no fallas de los negocios) (Parker et al., 2013: 1359).

Por estos motivos, mientras la comunidad internacional ("top-top-down”) y la presión social (bottom-up) no actúen, no es esperable que la élite tome medidas y realice los cambios necesarios para frenar el cambio climático, pues "los actores pueden comportarse estratégicamente encuadrando la escala del problema, situándose a sí mismos al centro del poder o evitando la responsabilidad por el problema escalándolo hacia arriba a hacia abajo" (Dewulf, 2013: 327).

Finalmente, el encuadre "medidas existentes", que contrariamente al de planificación se ocupa del cambio climático actual, no del proyectado (Juhola et al., 2011: 456), emerge enumerando acciones orientadas a contener los impactos en la agricultura ( 4 de 7 noticias), con un foco particular en la gestión de los recursos hídricos. Aun cuando el número de noticias es bajo en relación con la muestra (4/58), la tendencia apunta a que en Chile las políticas se están orientando hacia la agroindustria, especialmente la vitivinícola.

\section{La política como fuente primaria}

Los actores visibilizados en la muestra que tienden a constituirse como fuentes primarias de la misma son fundamentalmente políticos $(\mathrm{N}=23)$, representando un 67,6\%; más atrás siguen los empresarios $(\mathrm{N}=7)$ con un $20,5 \%$; y finalmente los científicos $(\mathrm{N}=4)$ con sólo un $11,8 \%$. No emergen actores ciudadanos en estas narrativas. Estas magras cifras de científicos reportando el cambio climático son consistentes con lo que sucede en Estados Unidos, donde "sus voces son frecuentemente marginalizadas en la arena de la política pública” (Alley, 2012: 178). 
Esta práctica de exclusión de la ciudadanía (personas y organizaciones) como fuentes informativas, es decir, con un rol activo en la construcción del debate en el espacio público informado por los medios de comunicación analizados, fractura "la relación dialógica que tendría que darse entre los actores de la comunicación política” (Reyes Montes, 2007: 129).

En un estudio del rol de la prensa en la construcción de representaciones ambientales en los habitantes de Saltillo, México, Carabaza (2007: 64) plantea que "las organizaciones civiles, los ciudadanos y los especialistas se encuentran marginados en la información, ya que son considerados esporádicamente como fuentes periodísticas importantes, tal vez esto se deba a la falta de estrategias de comunicación de estos grupos o a las rutinas profesionales que se les impone a los periodistas para la recolección de la información”.

Así, es la política quien define y encuadra el problema y sus soluciones (adaptación y mitigación), lo cual implica que los tomadores de decisión no sólo “toman las decisiones”, sino que construyen casi en solitario la discusión que finaliza en la decisión. Científicos y ciudadanos corrientes no poseen así el poder de influir en la construcción de agenda pública que sí les entregan los medios estudiados a la política, faltando así un contrapeso que nivele el poder de definición hacia actores distintos a los políticos y empresariales.

Un problema que surge de dicha posición dominante es el hecho de que las señales políticas en las noticias acerca del cambio climático "activa creencias ideológicas y hace de esas creencias predictores de preocupación mayor" que los proyectados por las élites científicas, ya sea existiendo o no un consenso (Alley, 2012: 178; Wiest et al., 2015: 194). Esto es relevante, ya que en la muestra estudiada para el caso chileno la gran mayoría de los actores políticos presentados son del gobierno o partidarios del gobierno de Sebastián Piñera. ${ }^{14}$

En los medios analizados, el riesgo que se presenta es que los actores visibilizados al poseer una tendencia política homogénea (derecha) teñirían ideológicamente la recepción del público, situación que debe ser remediada, tal como Giddens (2010) señala al apuntar a la necesidad de evitar convertir el cambio climático en capital político, por cuanto dificultaría el trabajo político en el largo plazo, así como dividiría a la opinión pública en un clivaje ideológico-político.

El proceso de "definición del problema" es relevante, pues construye la significancia social de la cuestión tratada, su sentido, implicancias y urgencia (Rochefort y Cobb, 1994: 28). El hecho de que la definición de un problema complejo sea realizada eminentemente por la política no es un problema en

14 Presidente de centro derecha (Partido Renovación Nacional), gobernó al país entre 2010 y 2014. 
sí, pues si existe un buen entendimiento del proceso científico podrían perfectamente traducir el amplio consenso científico expresado en el informe del IPCC, así como de las acciones requeridas de mitigación y adaptación.

Sterman y Booth-Sweeney (2007: 235) comprobaron que adultos con entrenamiento sustantivo en ciencia, tecnología, ingeniería y matemáticas (STEM) sufren sesgos sistemáticos en su juicio, decisiones y evaluaciones de las dinámicas del sistema climático, al hacer un ejercicio en estudiantes del MIT, ${ }^{15}$ tomando como referencia el Summary for Policy Makers del Tercer Informe de Evaluación del IPCC.

Desde esta perspectiva no es factible asumir que los actores políticos sean capaces de comprender y comunicar un proceso complejo como el cambio climático hacia las audiencias, dado que, en Estados Unidos, estos individuos son demográficamente similares a los líderes empresariales y gubernamentales, situación que no necesariamente concuerda con el perfil educativo de los actores políticos chilenos.

\section{Invisibilización de las personas y organizaciones sociales}

Apartar a las personas y las comunidades locales de los encuadres noticiosos genera: i) una mala comprensión -adquisición y empleo de conocimiento factual correcto-, ii) percepciones erradas - miradas e interpretaciones basadas en creencias y prejuicios- y iii) bajo compromiso - estado de conexión personal que incluye dimensiones cognitivas, afectivas y de comportamiento- de parte del público (Wolf y Moser, 2011: 548; Ford y King, 2015: 144). Esto es así, pues "las interpretaciones que da la audiencia [...] están manifiestamente constreñidas por lo que se informa, por lo que se omite y, tal vez más fundamentalmente, por las implicaciones de las noticias que establecen límites en la aptitud de los ciudadanos para influir en la política" (Edelman, 2002: 113).

Cuando las noticias invisibilizan a personas y comunidades sistemáticamente respecto a su rol frente al cambio climático, lo que en forma latente se está incubando es el desastre. Recordemos que éstos son eventos socialmente construidos, "producto del impacto de un peligro natural en personas cuya vulnerabilidad ha sido creada por condiciones sociales, económicas y políticas" (Cannon y Müller-Mahn, 2010: 622).

Esta asimetría de información entre los actores clave puede causar confusión en las audiencias, poniendo a las personas ante riesgos innecesarios (Aldunce et al., 2014b), situación que puede ser observada como "externalidades negativas", producto de narrativas que exacerban el rol de los tomadores 15 Massachusetts Institute of Technology. 
de decisiones y expertos, al utilizar enfoques como "de arriba a abajo" y "comando y control”. Como resultado de estas posturas mecanicistas, "un papel fundamental es dado a las agencias del gobierno y los profesionales en esta narrativa, que tiende a limitar el papel de las comunidades" (Aldunce et al., 2014a: 259).

\section{Conclusión}

Chile, como país de ingreso medio, vive tensiones internas entre sus élites político-económicas, que basan sus programas en un crecimiento económico con fuertes externalidades negativas para el medioambiente, y sectores ciudadanos que muestran signos de disposición a buscar formas alternativas de desarrollo, pero quizá esperando a que sus líderes instalen dichas opciones en el debate.

Este trabajo aporta evidencia empírica inédita en relación con los encuadres mediales para comunicar el cambio climático en Chile, utilizados por cuatro medios de prensa digital con altos niveles de lectoría y reconocimiento por parte de distintos actores del país: La Nación, El Mostrador, La Tercera y El Mercurio.

Un primer hallazgo general es que estas tensiones sobre modelos de desarrollo no son presentadas en los cuatro medios estudiados para el caso del cambio climático, favoreciendo, por lo tanto, la posición discursiva de las élites.

La manera en cómo es representado el cambio climático en los medios es clave para informar a las personas y comunidades acerca de sus deberes y derechos respecto a una adecuada provisión de Estabilidad Climática Global, ${ }^{16}$ y en caso de que dicho suministro sea escaso, cuáles son las acciones cotidianas que deben realizar para aportar a su cuidado, o en relación con cómo prepararse para actuar en caso de que fallen los planes diseñados para minimizar los peligros asociados.

Notamos así que la vulnerabilidad puede ser creada también por condiciones deficientes de circulación del conocimiento, capital cultural que permitiría la preparación y precaución, pero quizá en forma tan o más crucial que aquél, tener acceso a percepciones, valores y normas que, en definitiva, sostienen las visiones de mundo y los estilos de vida de las personas y sus comunidades coherentes con la trasformación hacia una civilización baja en carbono.

16 La Estabilidad Climática Global es el bien público global amenazado por el cambio climático. Office of Development Studies (ODS) (2002), Profiling the Provision Status of Global Public Goods. An ODS Staff Paper. United Nations Development Programme, New York. 
Los medios analizados, representantes de las tendencias políticas con acceso al poder en Chile hoy, son consistentes en alejar las acciones de mitigación y adaptación al cambio climático de las personas y el nivel local, minimizando el potencial que en ellos reside para cambiar el comportamiento de los sujetos, sus prácticas y estructuras sociales.

\section{Referencias}

Aldunce, Paulina et al. (2014a), "Framing disaster resilience. The implications of the diverse conceptualisations of 'bouncing back', en Disaster Prevention and Management, año 23, núm. 3, Reino Unido: Emerald.

Aldunce, Paulina et al. (2014b), "Resilience for disaster risk management in a changing climate: Practitioners' frames and practices", en Global Environmental Change, núm. 11, Reino Unido: Elsevier.

Alley, Kristen (2012), "Mass media roles in climate change mitigation", en Chen, Wei-Yin, Steiner, John, Suzuki, Toshio y Lackner, Maximilian [eds.], Handbook of Climate Change Mitigation, Alemania: Springer.

Anderson, Alison (2009), "Media, Politics and Climate Change: Towards a New Research Agenda", en Sociology Compass, núm 3, Estados Unidos: Wiley.

Arcila-Calderón, Carlos et al. (2015), "Media coverage of climate change in spanishspeaking online media”, en Convergencia. Revista de Ciencias Sociales, año 22, núm. 68, México: Universidad Autónoma del Estado de México.

Ayers, Jessica y Huq, Saleemul (2008), "The value of linking mitigation and adaptation: a case study of Bangladesh", en Environmental Management, año 43, núm. 5, Alemania: Springer.

Bassett, Thomas y Fogelman, Charles (2013), "Deja vu or something new? The adaptation concept in the climate change literature", en Geoforum, núm. 48, Reino Unido: Elsevier.

Baumgartner, Frank y Jones, Brian (1993), Agendas and instability in American politics, Estados Unidos: University of Chicago Press.

Boykoff, Maxwell y Boykoff, Jules (2004), "Balance as bias: global warming and the US prestige press", en Global Environmental Change, año 14, núm. 2, Reino Unido: Elsevier.

Boykoff, Maxwell (2007), "Flogging a dead norm? Newspaper coverage of anthropogenic climate change in the United States and United Kingdom from 2003 to 2006", en Area, año 39, núm. 4, Maiden (MA), Estados Unidos.

Boykoff, Maxwell y Boykoff, Jules (2007), "Climate change and journalistic norms: A case study of US mass-media coverage”, en Geoforum, núm. 38, Reino Unido: Elsevier.

Boykoff, Maxwell y Yulsman, Tom (2013), "Political economy, media, and climate change: sinews of modern life", en Wiley Interdisciplinary Reviews: Climate Change, año 4, núm. 5, Estados Unidos: Wiley.

Broomell, Stephen et al. (2015), "Personal experience with climate change predicts intentions to act”, en Global Environmental Change, núm. 32, Reino Unido: Elsevier.

Cannon, Terry y Müller-Mahn, Detlef (2010), "Vulnerability, resilience and development discourses in context of climate change", en Natural Hazards, vol. 55, núm. 3, Alemania: Springer. 
Julio Octavio Hasbún-Mancilla, Paulina Paz Aldunce-Ide, Gustavo Blanco-Wells y Rodrigo BrowneSartori. Encuadres del cambio climático en Chile: Análisis de discurso en prensa digital

Carabaza, Julieta (2007), "El papel de la prensa en la construcción de las representaciones sobre la problemática ambiental en los habitantes de Saltillo, Coahuila”, en Convergencia. Revista de Ciencias Sociales, año 8, núm 24, México: Universidad Autónoma del Estado de México.

Cervantes-Baraba, Cecilia (2001), "La Sociología de las Noticias y el Enfoque AgendaSetting”, en Convergencia. Revista de Ciencias Sociales, año 14, núm 43, México: Universidad Autónoma del Estado de México.

Chandler, Daniel y Munday, Rod (2011), Dictionary of media and communication, Reino Unido: Oxford University Press.

Consejo Nacional de la Cultura y las Artes (2012). Encuesta Nacional de Participación y Consumo Cultural. Disponible en: http://www.cultura.gob.cl/wp-content/ uploads/2013/11/ENPCC_2012.pdf [10 de marzo de 2017].

Dewulf, Art (2013), "Contrasting frames in policy debates on climate change adaptation”, en Wiley Interdisciplinary Reviews: Climate Change, año 4, núm. 4, Reino Unido: Wiley.

Edelman, Murray (2002), La construcción del espectáculo político, Argentina: Manantial.

Ford, James y King, Diana (2015), "Coverage and framing of climate change adaptation in the media: A review of influential North American newspapers during 1993-2013”, en Environmental Science \& Policy, año 48, Reino Unido: Elsevier.

Fraser, Evan et al. (2013), "Vulnerability hotspots: Integrating socio-economic and hydrological models to identify where cereal production may decline in the future due to climate change induced drought", en Agricultural and Forest Meteorology, año 170, núm. 15, Reino Unido: Elsevier.

Gandy, Oscar (1989), "The surveillance society: information technology and bureaucratic social control”, en Journal of Communication, año 39, núm. 3, Estados Unidos: Wiley.

Garreaud, René (2011), “Cambio Climático: Bases físicas e impactos en Chile”, en Revista Tierra Adentro (INIA-Chile), núm. 93. Disponible en: http://dgf.uchile.cl/rene/ PUBS/inia_RGS_final.pdf [20 de junio de 2015].

Giddens, Anthony (2010), La política del cambio climático, España: Alianza.

Hall, Stuart et al. (1978), "The social production of news", en Marris, Paul y Thornham, Susan (2000), Media Studies. A reader, Estados Unidos: New York University Press.

Hardoy, Jorgelina y Pandiella, Gustavo (2009), "Urban poverty and vulnerability to climate change in Latin America”, en Environment and Urbanization, año 21, Estados Unidos: Sage.

Human Rights Watch (1998), Los limites de la tolerancia. Libertad de expresión y debate público en Chile, Chile: LOMEdiciones.

IPCC (Intergobernmental Panel on Climate Change) (2014), Climate Change 2014: Impacts, Adaptation, and Vulnerability: IPCC Working Group II Contribution to AR5. Disponible en: http://ipcc-wg2.gov/AR5/ [20 de junio de 2015].

Jang, Mo y Hart, Sol (2015), "Polarized frames on 'climate change' and 'global warming' across countries and states: Evidence from Twitter big data”, en Global Environmental Change, núm. 32, Reino Unido: Elsevier.

Jogesh, Anu (2012), "A change in climate?: Trends in climate change reportage in the Indian print media”, en Dubash, Navroz [ed.], Handbook of Climate Change and India, Inglaterra: Earthscan.

Juhola, Sirkku et al. (2011), "Understanding the framings of climate change adaptation across multiple scales of governance in Europe", en Environmental Politics, año 20, núm. 4, Reino Unido: Routdledge. 
Convergencia Revista de Ciencias Sociales, núm. 74, 2017, Universidad Autónoma del Estado de México

Kingdon, John (1995), Agendas, alternatives and publicpolicies, Estados Unidos: Little, Brown. Mastrini, Guillermo y Becerra, Martín (2006), Periodistas y magnates: estructura y concentración de las industrias culturales en América Latina, Argentina: Instituto Prensa y Sociedad, Prometeo Libros.

Melo, Fabiola (2013) "Mineduc: más de 5 millones de chilenos mayores de 18 años aún no termina la educación escolar”, en La Tercera. Disponible en: http://www.latercera. $\mathrm{com} /$ noticia/mineduc-mas-de-5-millones-de-chilenos-mayores-de-18-anos-aun-notermina-la-educacion-escolar $\angle$ [ 10 de marzo de 2017].

Ockwell, Donald et al. (2009), "Reorienting Climate Change Communication for Effective Mitigation”, en Science Communication, año 30, núm. 3, Estados Unidos: Sage.

Olausson, Ulrika (2009), "Global warming--global responsibility? Media frames of collective action and scientific certainty", en Public Understanding of Science, año 18, núm. 4, Reino Unido: Sage.

Painter, James y Ashe, Teresa (2012), "Cross-national comparison of the presence of climate scepticism in the print media in six countries, 2007-10”, en Environmental Research Letters, año 7, Reino Unido: IOP Publishing.

Parker, Cristián et al. (2013), "Elites, climate change and agency in a developing society: the Chilean case", en Environment, Development and Sustainability, año 15, núm. 5, Alemania: Springer.

Pralle, Sarah (2009), “Agenda-setting and climate change", en Environmental Politics, vol. 18, núm. 5, Reino Unidos: Taylor and Francis.

Reyes Montes, María Cristina (2007), “Comunicación política y medios en México: el caso de la reforma a la Ley Federal de Radio y Televisión”, en Convergencia. Revista de Ciencias Sociales, vol. 14, núm. 43, México: Universidad Autónoma del Estado de México.

Rochefort, David y Cobb, Roger (1994), The politics of problem definition, Estados Unidos: University Press of Kansas.

Ryan, Michael et al. (1991), "Risk Information for Public Consumption: Print Media Coverage of Two Risky Situations", Health Education and Behavior, vol. 18, núm. 3, Estados Unidos: Sage.

Schmidt, Andreas et al. (2013), "Media attention for climate change around the world: a comparative analysis of newspaper coverage in 27 countries", en Global Environmental Change, 23, Reino Unido: Elsevier.

Somorin, Olufunso et al. (2012), "Congo basin forests in a changing climate: policy discourses on adaptation and mitigation (REDD+)", en Global Environmental Change, vol. 22, núm. 1, Reino Unido: Elsevier.

Steiner, Achim (2013), Director del Programa de Naciones Unidas para el Medio Ambiente (PNUMA), La Segunda online, 5 de noviembre de 2013. Disponible en: http:// www.lasegunda.com/Noticias/CienciaTecnologia/2013/11/890458/Advierten-quecontrolar-aumento-de-la-temperatura-de-la-tierra-se-hace-mas-dificil $[20$ de junio de 2015].

Sterman, John y Booth-Sweeney, Linda (2007), "Understanding public complacency about climate change: adults' mental models of climate change violate conservation of matter”, en Climatic Change, año 80, núm. 3-4, Alemania: Springer.

Sterman, John (2011), "Communicating climate change risks in a skeptical world", en Climatic Change, año 108, núm. 4, Alemania: Springer.

Sunkel, Guillermo y Geoffroy, Esteban (2001), Concentración económica de los medios de comunicación, Chile: LOM Ediciones. 
Julio Octavio Hasbún-Mancilla, Paulina Paz Aldunce-Ide, Gustavo Blanco-Wells y Rodrigo BrowneSartori. Encuadres del cambio climático en Chile: Análisis de discurso en prensa digital

Takahashi, Bruno y Meisner, Mark (2013), “Agenda Setting and Issue Definition at the Micro Level: Giving Climate Change a Voice in the Peruvian Congress", en Latin American Policy, año 4, núm. 2, Estados Unidos: Wiley.

Tankard, James (2001), “The empirical approach to the study of media framing”, en Reese, Stephen y Gandy, Oscar [eds.], Framing public life: Perspectives on media and our understanding of the social world, Estados Unidos: Routledge.

UDP-Feedback (2011), Primer estudio nacional sobre lectoría de medios escritos, Chile: Escuela de Periodismo Universidad Diego Portales.

Uusi-Rauva, Christa y Tienari, Janne (2010), "On the relative nature of adequate measures: Media representations of the EU energy and climate package”, en Global Environmental Change, año 20, Reino Unido: Elsevier.

Van Dijk, Teun (2002), "Critical discourse studies: a sociocognitive approach", en Wodak, R. y Meyer, M. [eds.], Methods of critical discourse analysis, Inglaterra: Sage.

Vicuña, Sebastián (2012), "Adaptation challenges for middle income countries: the experience of Chile”, en Centro de Cambio Global UC. Adaptation Futures. International Conference on Climate Adaptation (PPT), Estados Unidos.

Wiest, Sara et al. (2015), "Framing, partisan predispositions, and public opinion on climate change", en Global Environmental Change, núm. 31, Reino Unido: Elsevier.

Wolf, Johanna y Moser, Susanne (2011), "Individual understandings, perceptions, and engagement with climate change: insights from in depth studies across the world", en WIRES Climate Change, año 2, Estados Unidos: Wiley.

\section{Anexo}

Tabla 1

Medios digitales seleccionados

\begin{tabular}{lll}
\hline $\begin{array}{l}\text { Lectoría } \\
\text { Tendencia }\end{array}$ & \multicolumn{1}{c}{ + Leído } & \multicolumn{1}{c}{ - Leído } \\
& $\begin{array}{l}\text { www.emol.com } \\
\text { El sitio con mayor credibilidad y } \\
\text { el tercero más leído, luego de terra. } \\
\text { cl y lun.cl }\end{array}$ & $\begin{array}{l}\text { Sww.latercera.com } \\
\text { Segundo sitio con mayor } \\
\text { credibilidad y el cuarto más leído } \\
\text { en el país } \\
\text { Ediciones en formato impreso y } \\
\text { digital }\end{array}$ \\
& digital & www.lanacion.cl \\
Centro & www.elmostrador.cl & Nóptimo sitio más leído y el sexto \\
Izquierda & más creíble & $\begin{array}{l}\text { Noveno sitio de noticias de } \\
\text { internet más leído }\end{array}$ \\
& Sólo es editado digitalmente & Sólo es editado digitalmente \\
\hline
\end{tabular}

Fuente: Elaboración propia con base en UDP-Feedback (2011). 
Tabla 2

Actores por medio de prensa

\begin{tabular}{lccccc}
\hline \multicolumn{1}{c}{ Acto Medio } & EMOL & La Tercera & La & Nación & Total Actor \\
\hline Políticos & 13 & 7 & 5 & $25(38.4 \%)$ \\
Científicos y expertos & 4 & 12 & 4 & $20(30.7 \%)$ \\
$\begin{array}{l}\text { Organismos } \\
\text { Internacionales }\end{array}$ & 7 & 5 & 1 & $13(20 \%)$ \\
Empresa & 4 & 1 & 1 & $6(9.2 \%)$ \\
Otros & 1 & 0 & 0 & $1(1.5 \%)$ \\
Total Medio & 29 & 25 & 11 & $65(100 \%)$ \\
\hline
\end{tabular}

Fuente: Elaboración propia. $\mathrm{N}=58$ Noticias.

Julio Octavio Hasbún-Mancilla. Magíster en Gestión y Políticas Públicas por la Universidad de Chile. Profesor de la cátedra de Cambio Climático y Salud de la Facultad de Medicina de la Universidad de Chile, y tesista del Centro de Ciencia del Clima y la Resiliencia, CR2. Líneas de investigación: comunicación del cambio climático, semiótica política, gestión de la innovación en el sector público. Publicaciones recientes: Guevara, G. y Hasbún, J., "Innovación en Chile como aporte a la democratización de los servicios: análisis del caso de Gendarmería", en Revista de Estudios de Políticas Públicas, vol. 2, noviembre, Chile (2015); Pavez, D., De la O, M., Catril, R., Hasbún, J., "La innovación y su efecto en las personas: una aproximación desde la experiencia en Gendarmería de Chile”, en Revista de Estudios Criminológicos y Penitenciarios, vol. 19, julio, Chile (2015); Hasbún, J., "Juventudes Comunistas de Chile: Súmate a la Alegre Rebeldía. El panfleto como dispositivo de la marcha estudiantil", en Ponce de la Fuente, H. y Dalmasso, M.T. [eds.], Trayectos Teóricos en Semiótica, Chile: Universidad de Chile, LOM (2015).

Paulina Paz Aldunce-Ide. Ph.D. en Ciencias Sociales y Gestión de Recursos Naturales. Investigadora del Centro de Ciencia del Clima y la Resiliencia, CR2; Subdirectora del Programa de gestión de Riesgos CITRID y Profesora-Investigadora del Departamento de Ciencias Ambientales y Recursos Naturales Renovables, Facultad de Ciencias Agronómicas, Universidad de 
Chile. Líneas de investigación: dimensión social e institucional de los desastres socio-naturales y el cambio climático. Publicaciones recientes: Aldunce P., Borquez R., Adler C., Blanco G., Garreaud R., "Unpacking Resilience for Adaptation: Incorporating Practitioners' Experiences through a Transdisciplinary Approach to the Case of Drought in Chile", en Sustainability, vol. 8, núm. 9, Suiza: MDPI (2016); Aldunce P., Beilin R., Handmer J., Howden M., "Resilience for disaster risk management in a changing climate: practitioners' frames and practices", en Global Environmental Change, Reino Unido: Elsevier (2014); Aldunce P., Beilin R., Handmer J., Howden M., "Framing disaster resilience: the implications of the diverse conceptualisations of 'bouncing back"', en Disaster Prevention and Management, vol. 23, núm. 3 Reino Unido: Emerald (2014).

Gustavo Blanco-Wells. Doctor en Sociología del Desarrollo. Profesor Asociado del Instituto de Historia y Ciencias Sociales, Universidad Austral de Chile. Investigador Adjunto del Centro de Ciencia del Clima y la Resiliencia, CR2. Investigador Asociado del Centro de Investigación Dinámica de Ecosistemas Marinos de Altas Latitudes (IDEAL), Chile. Líneas de investigación: sociología del cambio ambiental global, estudios sociales de ciencia y tecnología, desarrollo territorial. Publicaciones recientes: Aldunce, P., Bórquez, R., Adler, C., Blanco, G., Garreaud, R., "Unpacking Resilience for Adaptation: Incorporating Practitioners' Experiences through a Transdisciplinary Approach to the Case of Drought in Chile", en Sustainability, vol. 8, núm. 9, Suiza: MDPI (2016); Hernando, M. y Blanco, G., “Territorio y Energías Renovables No Convencionales: Aprendizajes para la construcción de política pública a partir del caso de Rukatayo Alto, Región de Los Ríos, Chile", en Gestión y Politica Pública, vol. XXV, núm. 1, México: Centro de Investigación y Docencia Económicas, A.C. (2016); Blanco, G., Arce, A. y E. Fisher, "Becoming a Region, Becoming Global, Becoming Imperceptible: Territorialising Salmon in Chilean Patagonia”, en Journal of Rural Studies, vol. 42, Reino Unido: Pergamon Press Ltd (2015).

Rodrigo Browne-Sartori. Doctor en Comunicación. Profesor-Investigador del Instituto de Comunicación Social, Universidad Austral de Chile. Líneas de investigación: comunicación y cultura, periodismo intercultural y teorías de la comunicación. Publicaciones recientes: Ortiz de Zárate, A., Browne, R., "Meditaciones de Juan García del Río: un intelectual colombiano para el periodismo de Ambas Américas", en Revista Estudos IberoAmericanos, vol. 39, núm. 1, Brasil: Pontificia Universidade Católica do Rio 
Grande do Sul, Facultade de Filosofia e Ciencias Humanas (2014); Browne, R., Castillo, A. M., "Análisis Crítico del Discurso de la representación intercultural en la prensa chilena”, en Convergencia. Revista de Ciencias Sociales, vol. 20, núm.62, México: Universidad Autónoma del Estado de México (2014); Browne, R., Ortiz de Zárata, A. y Hurtado, M., "Exclusiones sexuales: la farmacopornografía como dispositivo fuera de género", en Revista Alpha, núm. 41, Chile: Universidad de Los Lagos (2014); Browne, R., Romero, P. y Monsalve, S., "La cobertura regional del movimiento estudiantil chileno 2011: prensa impresa y prensa digital en La Región de Los Ríos (Chile)", en Estudios del Mensaje Periodístico, núm. 2, vol. 21, España: Universidad Complutense de Madrid (2015).

Recepción: 3 de octubre de 2015.

Aprobación: 12 de septiembre de 2016. 